Check for updates

Cite this: RSC Adv., 2018, 8, 10794

Received 19th December 2017

Accepted 10th March 2018

DOI: $10.1039 / c 7 r a 13452 e$

rsc.li/rsc-advances

\section{LAPONITE® nanorods regulating degradability, acidic-alkaline microenvironment, apatite mineralization and MC3T3-E1 cells responses to poly(butylene succinate) based bio-nanocomposite scaffolds}

\author{
Liangchen Tang, (D) a Wu Wei, ${ }^{\mathrm{b}}$ Xuehong Wang, ${ }^{a}$ Jun Qian, ${ }^{a}$ Jianyou $\mathrm{Li}^{\text {*c }}{ }^{\mathrm{c}} \mathrm{Axiang} \mathrm{He},{ }^{\mathrm{d}}$ \\ Lili Yang, ${ }^{d}$ Xuesheng Jiang, ${ }^{c}$ Xiongfeng $\mathrm{Li}^{c}$ and Jie Wei ${ }^{\star a}$
}

Novel bio-nanocomposite scaffolds for bone tissue engineering were prepared by incorporation of LAPONITE $®$ (LAP) nanorods into poly(butylene succinate) (PBSu). The results showed that the scaffolds had well interconnected macroporous structures with macropore size in the range of 200-400 $\mu \mathrm{m}$ and porosity of around $70 \%$. In addition, the water absorption, degradability and apatite mineralization ability of the scaffolds were clearly enhanced with the increase of LAP content. Moreover, the degradation of LAP produced alkaline products, which neutralized the acidic degradable products of PBSu, and formed a weak alkaline microenvironment similar to a biological environment. Furthermore, the adhesion, proliferation and differentiation of MC3T3-E1 cells onto the scaffolds were significantly promoted with the increase of LAP content, in which the scaffold with $30 \mathrm{wt} \%$ LAP (sPL30) exhibited the best stimulation effect on the cells responses. The results suggested that the promotion of cells responses could be ascribed to the improvements of surface characteristics (including roughness, hydrophilicity, ions release and apatite formation, etc.) of the scaffolds. The sPL30 scaffold with excellent biocompatibility, bioactivity and degradability had great potential for applications in bone tissue engineering.

\section{Introduction}

LAPONITE® (LAP) is a synthetic silicate nanomaterial composed of nanoscale crystals, which has recently been developed as a new functional material in nanomedicine, namely for the diagnosis and treatment of diseases, as well as for regenerative medicine and tissue engineering..$^{1-3}$ LAP has been shown to readily degrade in the physiological environment giving rise to non-toxic and even bioactive products. ${ }^{2}$ The discovery that LAP is bioactive, itself capable of promoting osteogenic differentiation of human mesenchymal stem cells (hMSCs), resulted in the increase of research interest on its use in the context of bone tissue engineering/regeneration. ${ }^{3}$ Study has shown that LAP induced the osteogenic differentiation of

${ }^{a}$ Key Laboratory for Ultrafine Materials of Ministry of Education, East China University of Science and Technology, 130 Meilong Road, Shanghai 200237, China. E-mail: jiewei7860@sina.com; Tel: +86-021-64252745

${ }^{b}$ College of Materials Science \& Engineering, Nanjing Tech University, Nanjing 210009, China.E-mail: wwl_zzu@163.com

${ }^{c}$ Huzhou Center Hospital, Department Orthopedic, 198 Hongqi Rd, Huzhou 313000, China

${ }^{d}$ Second Mil. Med. Univ., Changzheng Hosp., Dep. Orthopaed Surg., Shanghai 200003, China the MC3T3-E1 cells by enhancing ALP activity, runt-related transcription factor 2 (RUNX2) transcript upregulation, bonerelated matrix protein deposition (osteocalcin and osteopontin), following by matrix mineralization. ${ }^{4,5}$

In previous studies, LAP has been incorporated into electrospun poly(lactic-co-glycolic acid) nanofibers scaffolds, which remarkably enhanced osteoblastic differentiation (alkaline phosphatase activity and osteocalcin secretion) of human mesenchymal stem cells (hMSCs) ${ }^{3,6}$ In addition, nanocomposite hydrogel of cross-linked poly(ethylene oxide) and LAP with the content ranging from 40 to $70 \mathrm{wt} \%$ were prepared, which significantly stimulated the adhesion, spreading, proliferation and differentiation of the MC3T3-E1 cells. ${ }^{7}$ Moreover, LAP bioceramic was developed, which clearly promoted the growth of rat mesenchymal stem cells, and repaired the bone defects of mice. ${ }^{8}$ Furthermore, LAP functionalized with arginine, lysine or leucine has been shown to promote human skin fibroblast proliferation, which highlighted the bioactive behavior of LAP in the process of wound healing, indicating that LAP might be applied as wound dressing in the regeneration of skin ailments and wounds. ${ }^{9}$ Therefore, as a bioactive nanomaterial, LAP with good biocompatibility and degradability 
might have a great potential for application in bone tissue engineering.

Over the past few decades, bio-nanocomposite scaffolds combining polymeric matrices and nanosized bioactive fillers (such as hydroxyapatite, bioglass, calcium phosphate, etc.) have been shown to have a great potential in regenerative medicine and tissue engineering, due to their ability to mimic the structural properties of native bone tissues..$^{10-13}$ The great research efforts for designing the ideal bio-nanocomposite scaffolds for repair and regeneration of damaged/diseased tissues have revealed the promise of polymer based bio-nanocomposite scaffolds, which exhibited superior biological properties for bone tissue engineering because allowed tailoring the desired bioactivity, degradation and resorption kinetics of the scaffolds. ${ }^{14-16}$ Moreover, nanosized bioactive fillers incorporated in the polymeric scaffolds offer the required osteoconductivity and biocompatibility features that are able to improve the cell adhesion, proliferation and differentiation, as well as, new bone tissue ingrowth into the scaffolds, and ultimately repair bone defects. ${ }^{12,17}$ Furthermore, the bio-nanocomposite scaffolds containing polymers and bioactive nanoparticles hold nanofeatured structures with improved properties, such as high surface area, fast degradation rate, enhanced hydrophilicity, bioactivity and mechanical properties that are a must for the appropriate cellular adhesion, proliferation and differentiation, and bone defects repair. ${ }^{18,19}$

Synthetic biodegradable polymers, such as, polycaprolactone, poly(lactic acid), poly(glycolic acid) and their copolymer, etc., have been widely applied to assemble bone tissue engineering scaffolds. ${ }^{17-20}$ As one of synthetically biodegradable polymers, poly(butylene succinate) ( $\mathrm{PBSu}$ ) is an ideal alternative to tissue engineering scaffolds because of its excellent biocompatibility, good processing ability, as well as nontoxic degradable products, etc. ${ }^{20-23}$ However, as a scaffold for bone tissue engineering applications, PBSu still has some disadvantage. Previous studies demonstrated that biomaterials with hydrophilicity could be favourable for cell responses, thus endowing hydrophobic PBSu with good hydrophilicity would promote the cell responses (cell attachment and spreading). ${ }^{24-26}$ In addition, slow degradation and biological inertness of PBSu may hinder the osteogenesis and new bone tissues ingrowth into the scaffold. ${ }^{27,28}$

As a novel nanomaterial with excellent biocompatibility, bioactivity and degradability, LAP is a source of inspiration for the design of the new biomedical materials with improved biological performance because LAP can elicit specific biological responses. ${ }^{4}$ In addition, tissue engineering scaffold is of crucial importance since it acts as temporary porous template for cell adhesion, proliferation, osteogenic differentiation and ultimately bone formation/defect repair. ${ }^{13}$ Therefore, the structural design of the scaffold often considers the factors (such as porosity, pore size and shape, interconnected pores, etc.) to facilitate desired bone regeneration. ${ }^{29}$ Therefore, in this study, bio-nanocomposite scaffolds were prepared by incorporation of bioactive LAP nanorods into PBSu using the simple method of solvent casting-particulate leaching. The effects of LAP on the surface morphology, water absorption, degradability and bioactivity of the PBSu/LAP bio-nanocomposite scaffolds were evaluated, and the cells responses to the scaffolds were also investigated by using MC3T3-E1 cells.

\section{Materials and methods}

\section{Preparation of scaffolds}

The PBSu/LAP composite scaffolds were prepared by a method of solvent casting-particulate leaching using $\mathrm{NaCl}$ particulates $(200-400 \mu \mathrm{m})$ as the porogen. Briefly, PBSu $\left(M_{\mathrm{w}}=8 \times 10^{4}\right.$, Anqing Hexing Chemical Co., Ltd., China) was dissolved in chloroform (Shanghai Lingfeng Chemical Reagent Co., Ltd., China) under stirring. Then LAP powders (Altana, Germany) was added into the above mentioned solution with continuous stirring to obtain the composite slurry. Afterwards, the $\mathrm{NaCl}$ particulates (weight ratio of composite to $\mathrm{NaCl}=1 / 10$ ) were added into the slurry following with $2 \mathrm{~h}$ of stirring, and then put into cylindrical molds ( $\Phi 12 \times 2 \mathrm{~mm})$ and compression molded under $4 \mathrm{MPa}$ to obtain the mixture sample. The NaCl particulates were then leached by immersing the sample in deionized water and air-dried for $12 \mathrm{~h}$. Finally, the PBSu/LAP composite scaffolds with $15 \mathrm{wt} \%$ of LAP (sPL15) and $30 \mathrm{wt} \%$ of LAP (sPL30) were prepared. The PBSu scaffolds without LAP were also prepared according to the above mentioned procedure as a control (sPLO).

\section{Characterization of LAP and scaffolds}

The morphology, phase, composition and structure of LAP and PBSu/LAP composites was characterized by transmission electron microscopy (TEM, JEM-2010, JEOL), energy dispersive spectrometry (EDS, JEOL-6360LV, JEOL), wide-angle X-ray powder diffraction (XRD, D/max 2550 VB/PC, Rigaku Co., Ltd.) and fourier transformed infrared spectroscopy (FTIR, Magna-IR 550, Nicolet). The surface morphology of composite scaffolds was characterized by synchrotron radiation-based microcomputed tomography (SRmCT, Shanghai Synchrotron Radiation Facility) and scanning electron microscopy (SEM, S-3400N, Hitachi).

\section{Porosity and water adsorption}

The total porosity of scaffolds was determined by the method of ethanol substitution..$^{30}$ Porosity was calculated according to equation:

$$
\text { Porosity }(\%)=\left(V_{\mathrm{t}}-V_{\mathrm{a}}\right) / V_{\mathrm{t}} \times 100 \%
$$

where the total volume $\left(V_{\mathrm{t}}\right)$ of the scaffold was determined by measuring radius and height, and $V_{\mathrm{a}}$ represents volume of the scaffold immersed in ethanol.

The water absorption was evaluated as the difference between the weight of the samples immersed in water for $24 \mathrm{~h}$ $\left(M_{\mathrm{w}}\right)$ and the weight of the dry samples $\left(M_{\mathrm{d}}\right)$ according to equation:

$$
\text { Water absorption }(\%)=\left(M_{\mathrm{w}}-M_{\mathrm{d}}\right) / M_{\mathrm{d}} \times 100 \%
$$




\section{In vitro degradability}

To assess the degradability of the composite scaffolds (sPL0, sPL15 and sPL30), the weight loss ratio of the scaffolds and $\mathrm{pH}$ changes of the solution after soaked in Tris-HCl solution for different time were monitored. Briefly, the scaffolds were placed in separate polypropylene vials and incubated in the Tris- $\mathrm{HCl}$ solution ( $\mathrm{pH} \mathrm{7.4)}$ at $37{ }^{\circ} \mathrm{C}$. The scaffolds were taken out at the target intervals, and dried at $37{ }^{\circ} \mathrm{C}$. The weight loss of the scaffolds was calculated according to equation:

$$
\text { Weight } \operatorname{loss}(\%)=\left(m_{0}-m_{\mathrm{t}}\right) / m_{0} \times 100 \%
$$

Where $m_{0}$ represent the initial weight of the samples and $m_{\mathrm{t}}$ represent the weight of the samples after immersion in Tris$\mathrm{HCl}$ solution for different time.

The changes of $\mathrm{pH}$ of the Tris- $\mathrm{HCl}$ solutions in the samples soaked for different time were determined by a pH meter (PHS3C, INESA).

\section{In vitro bioactivity}

In vitro bioactivity of the composites scaffolds was determined by testing the apatite formation after the samples soaked into simulated body fluid (SBF, pH 7.4) at $37{ }^{\circ} \mathrm{C}$ with the solution volume/sample weight ratio of $20 \mathrm{~mL} \mathrm{~g}^{-1} .^{16}$ After soaking for different time, the samples were taken out and gently rinsed with deionized water, then dried in an oven at $50{ }^{\circ} \mathrm{C}$ for $24 \mathrm{~h}$. The surface morphology of the samples was determined by SEM, and the element composition of the deposits on samples surfaces was determined by EDS. The ion concentrations ( $\mathrm{Si}, \mathrm{Ca}$, $\mathrm{Mg}, \mathrm{P}$ and $\mathrm{Li}$ ions) in the SBF solution after the sPL30 soaked for $0.25,0.5,1,3$ and $7 \mathrm{~d}$ were determined by inductively coupled plasma atomic emission spectroscopy (ICP-AES, IRIS 1000, Thermo Elemental, USA).

\section{In vitro cytocompatibility of scaffolds}

Mouse pre-osteoblastic cell lines (MC3T3-E1) obtained from Shanghai Key Laboratory of Orthopaedic Implants, Shanghai Ninth People's Hospital (China) were cultured in DMEM (Thermo Fisher Scientific Ind, USA) supplemented with 10\% Fetal Bovine Serum (Gibco BRL, FBS), penicillin (100 $\left.\mathrm{U} \mathrm{mL}^{-1}\right)$ and streptomycin sulphate $\left(100 \mu \mathrm{g} \mathrm{mL} \mathrm{m}^{-1}\right)$ under a humidified atmosphere at $37{ }^{\circ} \mathrm{C}$ and $5 \% \mathrm{CO}_{2}$. The culture medium was replaced every $3 \mathrm{~d}$.

Cell morphology. All scaffolds $(\Phi 12 \times 2 \mathrm{~mm})$ were sterilized by ethylene oxide for subsequent cell experiments. The samples were placed in 24-well plates and seeded with MC3T3-E1 cells with a density of $1 \times 10^{5}$ cells per well and incubated at $37^{\circ} \mathrm{C}$. After $3 \mathrm{~d}$ of cultivation, the samples were washed with phosphate buffered saline (PBS) solution for 3 times, and the cells on the samples surfaces were fixed with $4 \%$ glutaraldehyde for $2 \mathrm{~h}$. Subsequently, cells were stained with fluorescein isothiocyanate (Sigma, FITC) for $40 \mathrm{~min}$ and 4',6-diamidino-2-phenylindole (Sigma, DAPI) for $5 \mathrm{~min}$, respectively. The actin filament and cell nuclei were observed by confocal laser scanning microscopy (CLSM, Nikon A1R, Japan). On the other hand, cells for the SEM observation were dehydrated with a series of ethanol solutions with different concentration. ${ }^{31}$ The morphology of cells on the samples was observed by SEM.

Cell proliferation. The proliferation of MC3T3-E1 cells was determined by using MTT-assay (3-(4,5-dimethylthiazol-2-yl)2,5-diphenyltetrazolium bromide) after 1,3 , and $5 \mathrm{~d}$ of culturing. ${ }^{32}$ Briefly, the samples were placed in 24-well plates and seeded with MC3T3-E1 cells with a density of $1 \times 10^{5}$ cells per well and incubated at $37{ }^{\circ} \mathrm{C}$. At each pre-determined time, the samples were placed into another 24 -well plates, $100 \mu \mathrm{L}$ of the MTT solution $\left(0.5 \mathrm{mg} \mathrm{mL}^{-1}\right)$ was added into the medium, and the samples were incubated at $37^{\circ} \mathrm{C}$ for $4 \mathrm{~h}$. After incubation, dimethyl sulfoxide (Sinopharm, DMSO) was added into the medium to dissolve the purple formazan. The solution was then incubated at $37{ }^{\circ} \mathrm{C}$ for another $30 \mathrm{~min}$, and the optical density (OD) was measured at the wavelength of $490 \mathrm{~nm}$ using a Microplate Reader (AMR-100, Allsheng Co., Ltd.,).

Alkaline phosphatase (ALP) activity. The ALP activity of MC3T3-E1 cells on the samples was tested by pNPP assay ( $p$ nitrophenyl-phosphate) at day 7, 10 and 14 after culturing. ${ }^{32} \mathrm{~A}$ total of $1 \times 10^{5}$ of cells were seeded on each samples in the 24 well plates. In this process, after aspirating the culture media in 24-well plates, cell lysate was obtained by adding $1 \%$ Nonidet P40 (NP-40) for $1 \mathrm{~h}$. Then, the supernatant was added into the 96well plates, which followed by adding pNPP (Sigma, USA) substrate solution composed of $0.1 \mathrm{M}$ glycine and $1 \mathrm{mM}$ $\mathrm{MgCl} \cdot 6 \mathrm{H}_{2} \mathrm{O}$. Finally, after incubating for $30 \mathrm{~min}$ at $37{ }^{\circ} \mathrm{C}$, the reaction was stopped by adding $0.1 \mathrm{M} \mathrm{NaOH}$ solution. The ALP activity was measured at $405 \mathrm{~nm}$ using a Microplate Reader. The total protein content in cell lysate was determined using the bicinchoninic acid method in aliquots of the same samples with the Pierce protein assay kit (Pierce Biotechnology Inc.,), read at $562 \mathrm{~nm}$ and calculated according to a series of albumin (bovine serum albumin) standards. The ALP activity was normalized to the total protein content.

Ions release from scaffolds in cell culture medium. To investigate the changes of ions concentrations in cell culture medium during the culture of MC3T3-E1 cells with samples, the cell culture medium for cell proliferation test was collected at day 1, 3 and 5, and the concentrations of $\mathrm{Mg}$, $\mathrm{Si}$ and $\mathrm{Li}$ ions from the scaffolds were detected by ICP-AES. The ions concentrations in DMEM without samples were also measured as the blank group.

\section{Statistical analysis}

Statistical analyses of the data were performed by using the SPSS15.0 software (SPSS, USA). The measurement data expressed as the mean \pm standard deviation for $n=3$, the data were analyzed by $t$ test. $p<0.05$ was considered significant differences, with statistical significance.

\section{Results}

\section{Characterization of scaffolds}

Fig. 1A is the TEM micrograph of LAP, it can be seen that LAP are short rod-like particles with the sizes of $10 \mathrm{~nm}$ in diameter 

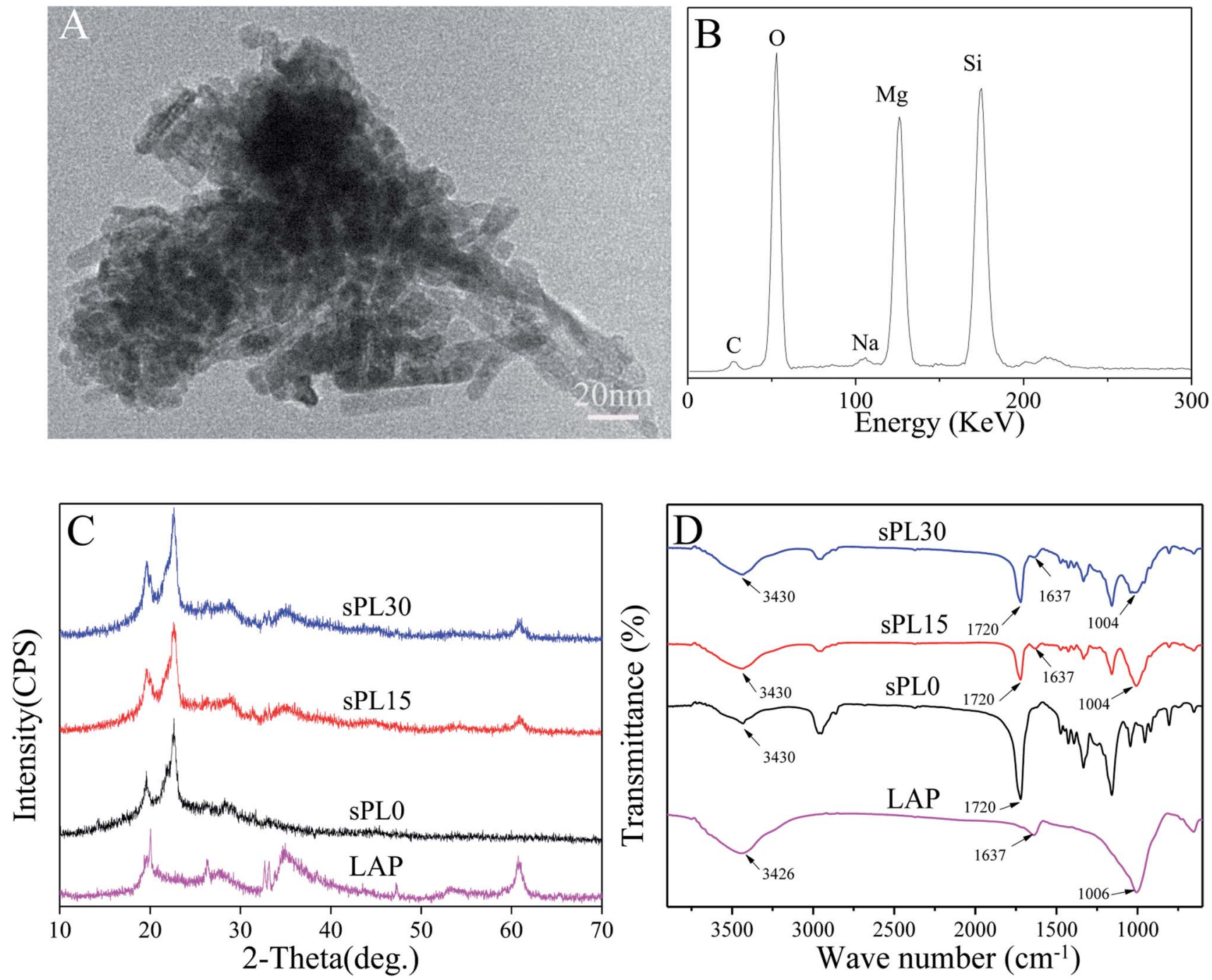

Fig. 1 TEM micrograph (A) and EDS (B) of LAP, and XRD (C) and FTIR (D) of LAP, sPLO, sPL15 and sPL30 scaffolds.

and $20-50 \mathrm{~nm}$ in length. Fig. 1B is the EDS of LAP, and the peaks of $\mathrm{Si}, \mathrm{Mg}$ and $\mathrm{O}$ elements were found.

Fig. 1C is XRD of LAP, SPL0, sPL15 and sPL30 scaffolds. It was found that the characteristic peaks of LAP were at $20^{\circ}, 35^{\circ}$ and $61^{\circ}$ while the characteristic peaks of sPL0 (PBSu) scaffold were at $19.5^{\circ}, 22.8^{\circ}$ and $28.2^{\circ}$. Moreover, the characteristic peaks of LAP and PBSu were found in both SPL15 and SPL30 scaffolds.

Fig. 1D is FTIR of LAP, SPL0, SPL15 and SPL30 scaffolds. The characteristic peaks of LAP appeared at $1006 \mathrm{~cm}^{-1}, 1637 \mathrm{~cm}^{-1}$ and $3426 \mathrm{~cm}^{-1}$, which are typical bands of $\mathrm{Si}-\mathrm{O}-\mathrm{Si}$ asymmetric stretching of silicate, structural hydroxyls and absorbed water, respectively. The peaks at $1720 \mathrm{~cm}^{-1}$ and $3430 \mathrm{~cm}^{-1}$ of SPLO correspond to the $\mathrm{C}=\mathrm{O}$ stretching and free $\mathrm{O}-\mathrm{H}$ groups of $\mathrm{PBSu}$, respectively. Furthermore, it was evident that the peaks of LAP and PBSu were presented in both SPL15 and SPL30 scaffolds.

Fig. 2(a-c) are the digital photos of sPL0 (a), sPL15 (b) and sPL30 (c) scaffolds. All the scaffolds showed a reticular structure without a clear visual difference in macroscopic size. Fig. 2(d-f) are the 2D cross-section images from SRmCT of SPL0 (d), SPL15 (e) and sPL30 (f) scaffolds, which showed well-interconnected pore structure. Fig. $2(\mathrm{~g}-\mathrm{i})$ are the $3 \mathrm{D}$ reconstructed images from SRmCT of SPL0 (g), SPL15 (h) and sPL30 (i) scaffolds, which revealed a highly porous structure.

Fig. 3 shows SEM micrographs of surface morphology of SPL0, sPL15 and sPL30 scaffolds. The macropore sizes of scaffolds were found to vary from 200 to $400 \mu \mathrm{m}$, which were consistent with the size of $\mathrm{NaCl}$ particles used to prepare the scaffolds. No clear difference in the macropore sizes of the scaffolds was observed for the three kinds of samples after introduction of LAP. In the high magnification of SEM micrographs, it was found that the SPL0 scaffolds showed a relatively smooth surface while the sPL15 and sPL30 scaffolds surface revealed rough surfaces.

The porosity and water absorption of the scaffolds are showed in Table 1. All samples exhibited high porosity, and the average porosity of sPL0, sPL15 and sPL30 scaffolds were 70.4\%, 

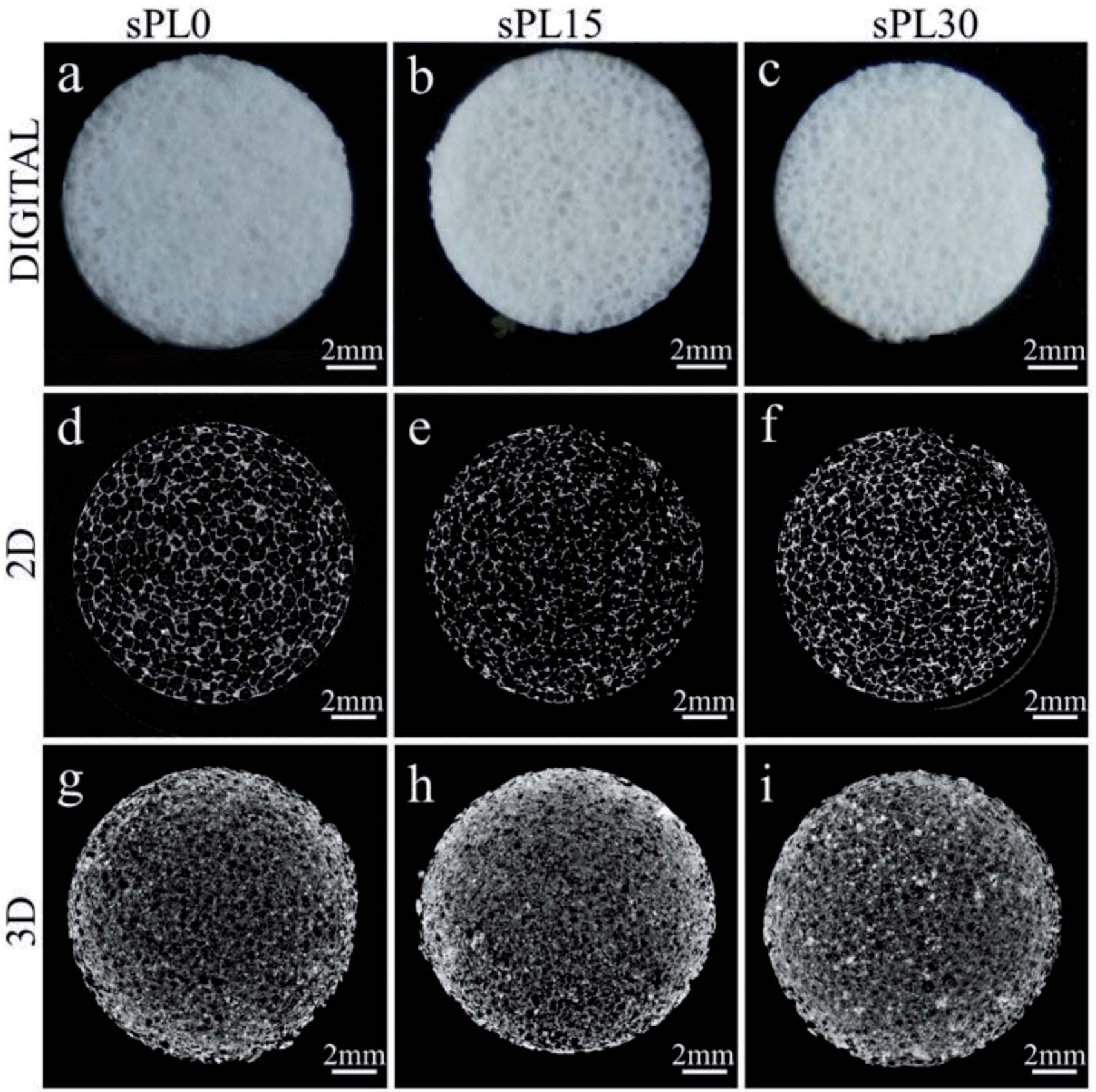

Fig. 2 Digital photos $(a-c)$, cross-section of 2D $(d-f)$ and 3D reconstructed images $(g-i)$ from SRmCT of sPL0, sPL15 and sPL30 scaffolds $(\Phi 12 \times 2 \mathrm{~mm})$.

$71.2 \%$ and $72.5 \%$, respectively. Moreover, the water absorption ratio of sPL0, sPL15 and sPL30 scaffolds were $335.2 \%, 360.4 \%$ and $397.6 \%$, respectively.

\section{Degradability of scaffolds in Tris-HCl solution}

Fig. 4A reveals the weight loss of SPL0, sPL15 and SPL30 scaffolds soaked into Tris-HCl solution for different time. It was found that the weight loss of the scaffolds in the solution increased with time, and the weight loss of the scaffolds increased with LAP content in composite scaffolds. At 84 days, the weight loss of sPL0, sPL15 and sPL30 were $16.8 \mathrm{wt} \%$, $34.2 \mathrm{wt} \%$ and $52.3 \mathrm{wt} \%$, respectively.

Fig. 4B shows the changes in $\mathrm{pH}$ of the Tris- $\mathrm{HCl}$ solutions in which sPL0, sPL15 and sPL30 scaffolds soaked for different time. It was found that $\mathrm{pH}$ for SPL0 decreased slowly from 7.4 to 6.9 during the whole immersion period. As for SPL15 and SPL30, $\mathrm{pH}$ increased in the first 21 days and then showed a slow decrease in the following 63 days. At day 21, $\mathrm{pH}$ of solutions containing sPL15 and sPL30 scaffolds were 7.56 and 7.71, respectively. Subsequently, pH for sPL15 and sPL30 scaffolds solutions stabilized around 7.4. 


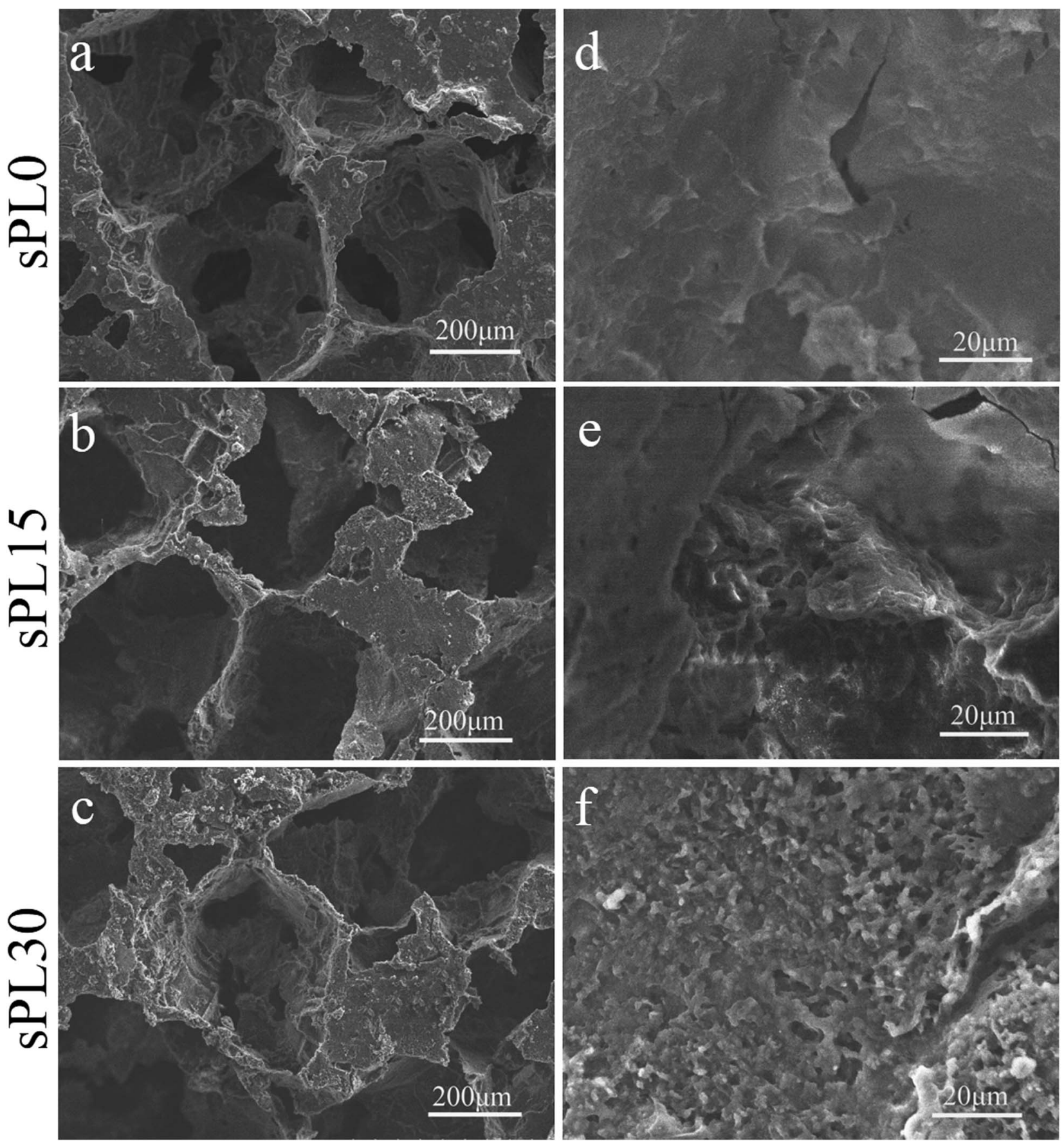

Fig. 3 SEM micrographs of surface morphology of sPLO (a and d), sPL15 (b and e) and sPL30 (c and f) scaffolds.

\section{Apatite formation on scaffolds in SBF}

The SEM micrographs of surface morphology of the SPL0, SPL15 and SPL30 scaffolds after immersion into SBF for 7 days are showed in Fig. 5. After 7 days of immersion in SBF, no apatite precipitate was found on the surface of SPL0 scaffold. However, a large number of apatite precipitates were found on the surfaces of SPL15 and SPL30 scaffolds. Moreover, the sPL30 were almost fully covered by apatite, which were more than sPL15 scaffolds.
Fig. 6A shows the EDS of the surface of sPL30 scaffold immersed into SBF for 7 days. The peaks of $\mathrm{Ca}$ and $\mathrm{P}$ elements were observed, and the ratio of $\mathrm{Ca}$ to $\mathrm{P}(\mathrm{Ca} / \mathrm{P}$ ratio) was 1.65 ,

Table 1 Porosity, water absorption of the scaffolds

\begin{tabular}{lllll}
\hline & & & & \multicolumn{2}{l}{$\begin{array}{l}\text { Water } \\
\text { absorption (\%) }\end{array}$} \\
\hline 100 & 0 & SPL0 & $70.4 \pm 3.3$ & $335.2 \pm 5.3$ \\
85 & 15 & SPL15 & $71.2 \pm 2.8$ & $360.4 \pm 12.3$ \\
70 & 30 & sPL30 & $72.5 \pm 3.5$ & $397.6 \pm 21.5$
\end{tabular}



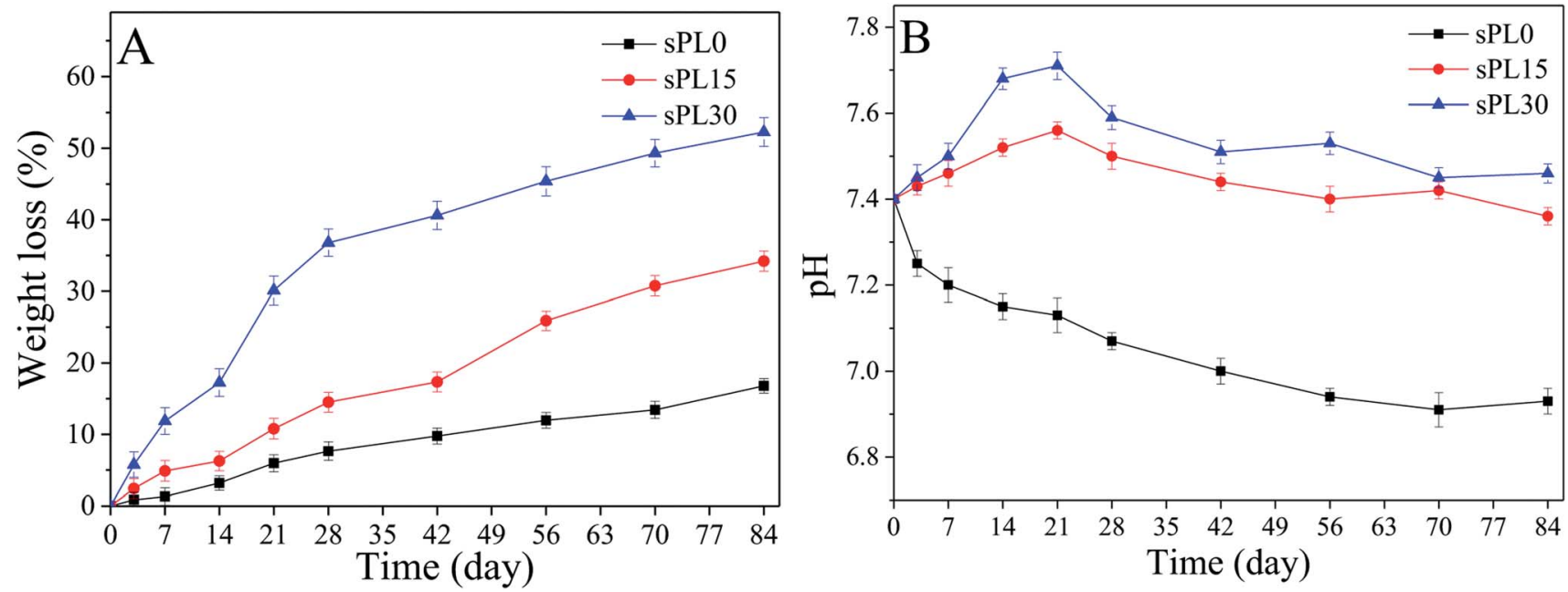

Fig. 4 Weight loss (A) of the samples and pH changes of solution (B) after sPLO, sPL15 and sPL30 scaffolds soaked into Tris-HCl solution for different time.

which was closed to the apatite $(\mathrm{Ca} / \mathrm{P}=1.5-1.67)$ in natural bone minerals. ${ }^{33}$ The changes of concentrations of $\mathrm{Ca}, \mathrm{Si}, \mathrm{P}, \mathrm{Li}$ and $\mathrm{Mg}$ ions in the SBF solution after sPL30 scaffold were soaked for different times were revealed in Fig. 6B. It was found that the concentration of $\mathrm{Mg}, \mathrm{Li}$ and $\mathrm{Si}$ ions gradually increased while $\mathrm{Ca}$ and $\mathrm{P}$ ions reduced with time.

\section{Cell morphology on scaffolds}

The SEM micrographs of morphology of MC3T3-E1 cells cultured on SPL0, sPL15 and SPL30 scaffolds at 3 days are shown in Fig. 7(a-c). Furthermore, CLSM images of cytoskeleton of MC3T3-E1 cells on the scaffolds stained by FITC and DAPI are shown in Fig. 7(d-f). FITC staining showed cytoplasmic for each cell (green) and DAPI staining showed round cell nucleus for each cell (blue). As shown in Fig. 7, at 3 days, only a small amount of cells attached on SPL0 and mostly in round appearance while some cells stretched to more regions, tightly adhered to the pore surface of SPL15, and exhibited long spindle polar alignment. For the sPL30, the cells showed excellent adhesion, stretching, and aggregation on the
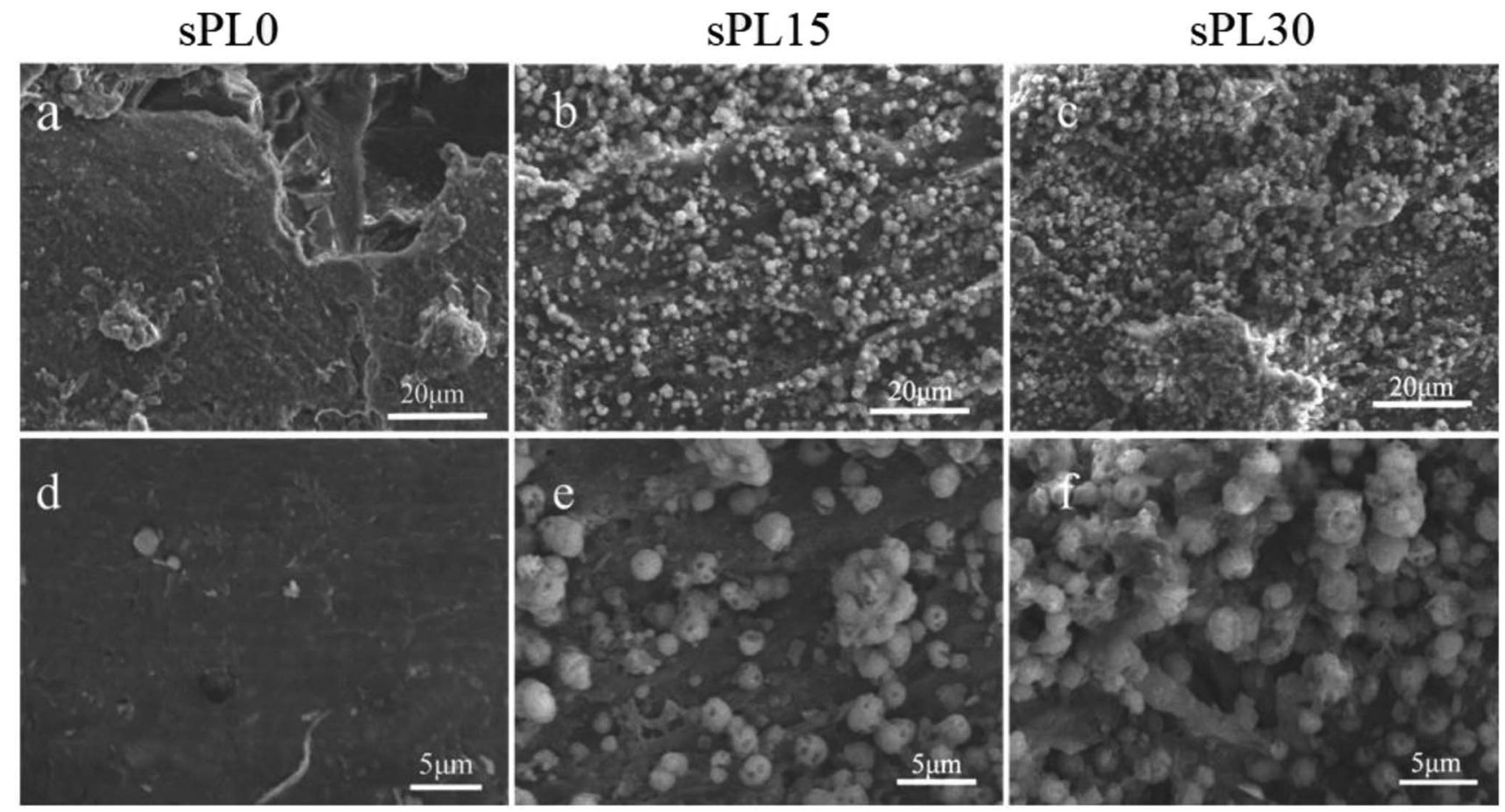

Fig. 5 SEM micrographs of surface morphology of sPLO (a and d), sPL15 (b and e) and sPL30 (c and f) scaffolds after immersion into SBF for 7 days. 

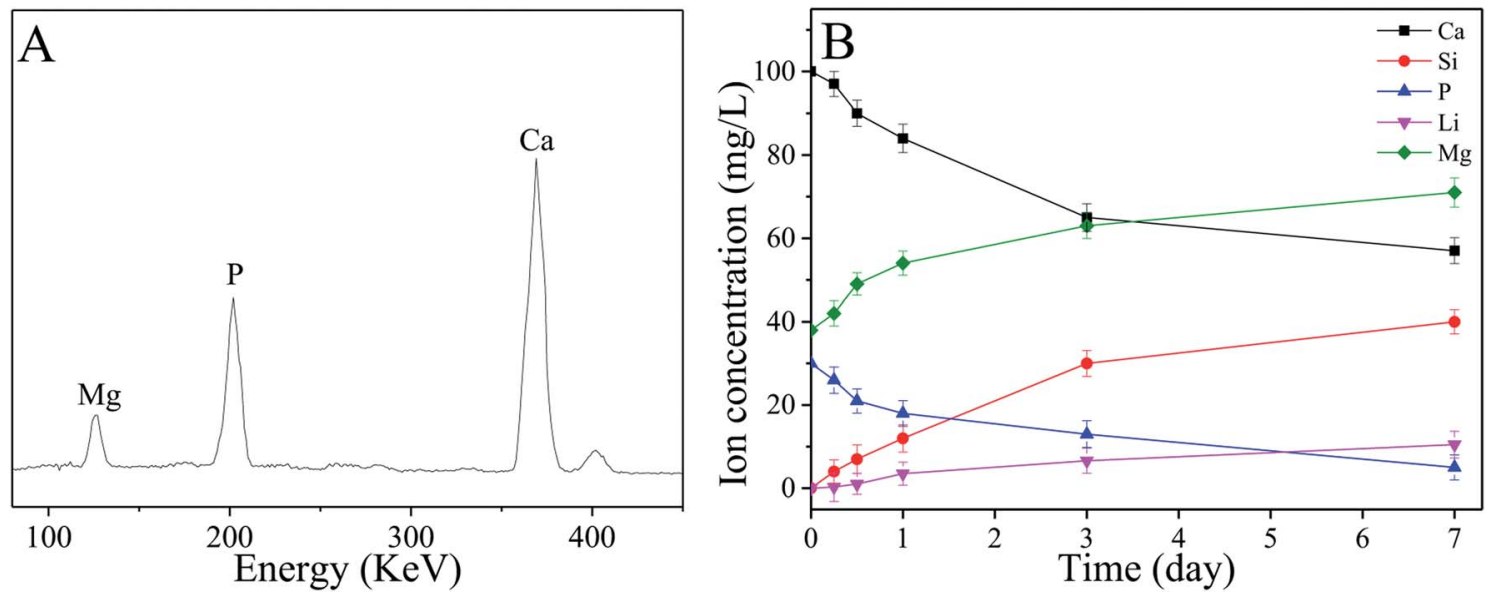

Fig. 6 EDS (A) of the surface of sPL30 scaffolds after immersion in SBF for 7 days and changes in ions concentrations of the SBF solution (B) for different time SPL30 incubation.

scaffolds, and exhibited the typical long spindle shape. The results indicated that the cells spread better on the sPL15 and sPL30 than SPL0 scaffolds.

\section{Cell proliferation and ALP activity on scaffolds}

The OD values (cell proliferation) of MC3T3-E1 cells cultured on the scaffolds are shown in Fig. 8A. On day 3 and 5 , the OD value of cells on sPL30 were clearly higher than sPL15 scaffolds, and
SPL15 was significantly higher than sPL0 scaffolds $(p<0.05)$. The results showed that composite scaffolds containing LAP clearly promoted the proliferation of MC3T3-E1 cells, which depended on LAP content.

The ALP activity (cell differentiation) of MC3T3-E1 cells cultured on the scaffolds is shown in Fig. 8B. On day 10, the ALP activity of cells on SPL30 was clearly higher than SPL15 and SPL0 scaffolds. Moreover, on day 14, the ALP activity of cells on sPL30
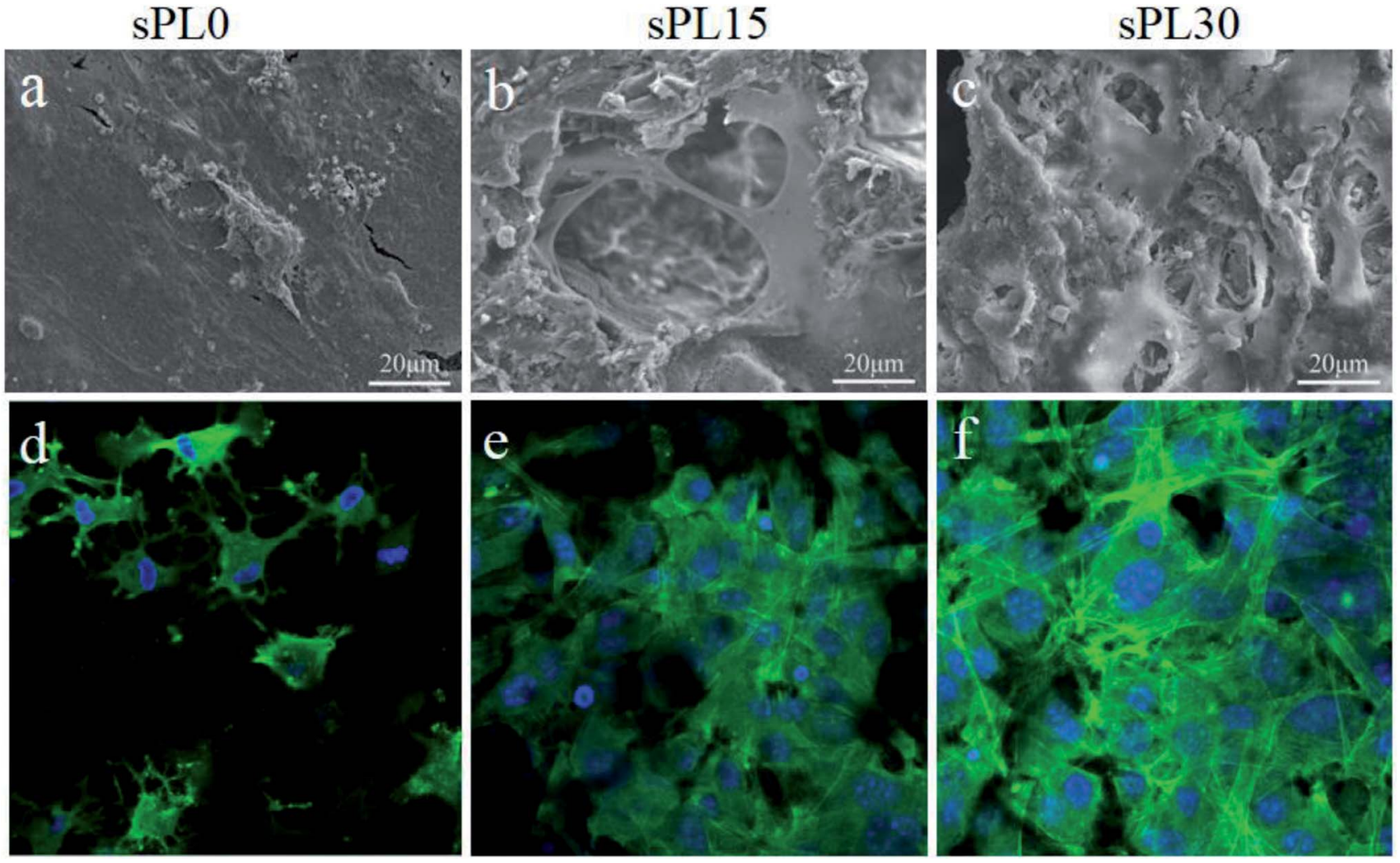

Fig. 7 SEM (a-c) micrographs and CLSM (d-f) images of MC3T3-E1 cells cultured on sPLO (a and d), SPL15 (b and e), sPL30 (c and f) scaffolds at 3 days. 

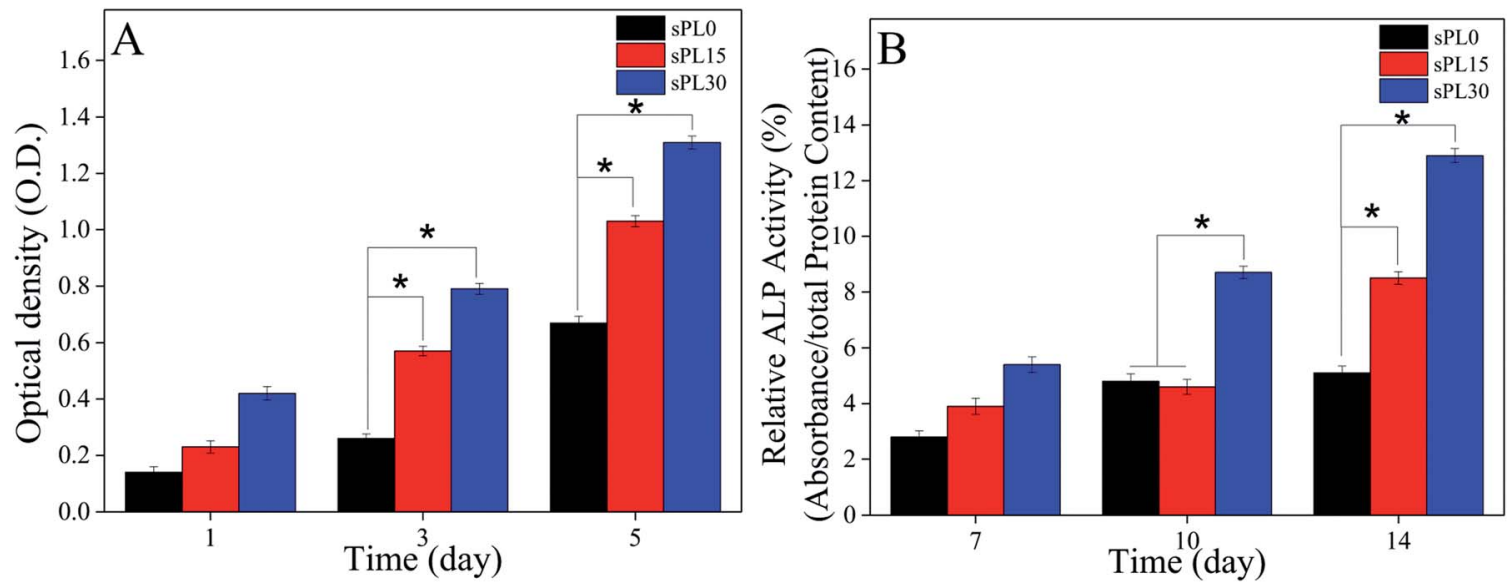

Fig. 8 OD values (A) and ALP activity (B) of MC3T3-E1 cells cultured on sPL0, sPL15 and sPL30 scaffolds for different times (*p < 0.05).

was clearly higher than sPL15 scaffolds, and the sPL15 was clearly higher than sPL0 scaffold $(p<0.05)$.

\section{Ions release from scaffolds into cell culture medium}

Fig. 9 reveals the changes of concentrations of $\mathrm{Mg}, \mathrm{Si}$ and $\mathrm{Li}$ ions in cell culture medium with times after the MC3T3-E1 cells cultured on the samples, including blank, sPL0, sPL15 and SPL30. The concentrations of $\mathrm{Mg}, \mathrm{Si}$ and $\mathrm{Li}$ ions in cell culture medium for both sPL15 and sPL30 scaffolds gradually increased with time because these ions gradually released from the two scaffolds into the medium. Moreover, these ions concentrations in medium for sPL30 were higher than sPL15 scaffold. No Li and Si ions were found in the cell culture medium of blank group and sPL0 scaffolds during the samples soaking time, and no significant difference of $\mathrm{Mg}$ ion concentrations were found in the blank group and sPL0 scaffolds.

\section{Discussion}

In this study, the PBSu/LAP bio-nanocomposite (SPL) scaffolds were prepared by incorporation of LAP nanorods into PBSu using the simple method of solvent-casting and particulateleaching. From the $2 \mathrm{D}$ cross-section and $3 \mathrm{D}$ reconstructed images by SRmCT, the scaffolds (SPL0, SPL15 and SPL30) exhibited well interconnected macroporous structure with the macropore size in the range from 200 to $400 \mu \mathrm{m}$. In addition, the scaffolds possessed high porosity with the average porosity of SPL0, SPL15 and sPL30 scaffolds were 70.4\%, 71.2\% and $72.5 \%$, respectively. No clear differences in macropore size of these scaffolds were observed, indicating that incorporation of LAP nanorods into PBSu had no clear effects on their pores sizes. Moreover, due to the incorporation of LAP nanorods into PBSu matrix, the surfaces of the composites scaffolds (sPL15 and SPL30) became rough as compared with PBSu (sPL0) scaffolds with the relatively smooth surface.

The water absorption of the scaffold is an indicator of hydrophilicity, which is beneficial to cells adhesion and proliferation on the scaffold surface, and also has important effects on the degradability of the scaffold. ${ }^{34}$ In this study, the water absorption of the scaffolds clearly increased with the increase of LAP content, and the sPL30 scaffolds obtained the highest water absorption among samples. The results indicated that the water absorption of the scaffolds was greatly enhanced by incorporation of LAP conferring higher hydrophilicity to PBSu matrix.

The degradation rate and by-products of the biodegradable scaffolds are critical in the role of bone regeneration..$^{35}$ Indeed, the degradation rate of a scaffold is slow, which may hinder new bone formation/ingrowth into porous structure and ultimately repair bone defects. ${ }^{22}$ In addition, the by-products upon degradation may alter the tissue micro-environment and then challenge the biocompatibility of the scaffold, and the subsequent tissue repair. ${ }^{35}$ In this study, the results showed that the degradation rate of the scaffolds in Tris-HCl solution were clearly improved with the increase of LAP content (sPL30 > SPL15 $>$ sPL0). The increase of degradation rate of the SPL30 scaffolds was ascribed to not only the degradation of LAP but also improved degradation of PBSu due to favored entrance of water molecules, which might accelerate the degradation of PBSu.

Previous study showed that the by-products of PBSu degradation contained succinic acid, leading to acidic microenvironment, which might be prone to aseptic inflammation in the body. ${ }^{17}$ In this study, $\mathrm{pH}$ of the solution for sPL0 decreased slowly from 7.4 to 6.9 during the whole immersion period, indicating that the degradation of PBSu produced acidic products. As for sPL15 and sPL30, pH increased from 7.4 to 7.56 and 7.71 in the first 21 days and then showed a slow decrease and stabilized around 7.4 in the following 63 days. The results indicated that the degradation of LAP produced alkaline products, which could neutralize the acidic by-products of PBSu degradation, and formed a weak alkaline environment $(\mathrm{pH}=$ 7.4) similar to biological environment. Therefore, it can be suggested that the scaffolds containing LAP can be considered as an advantage to avoid possible inflammatory response caused by acidic products of PBSu degradation, which may be very useful for cell growth/bone formation in vivo.

Apatite mineralization on the biomaterial surface in SBF is a well-accepted method to evaluate the in vitro bioactivity, which 

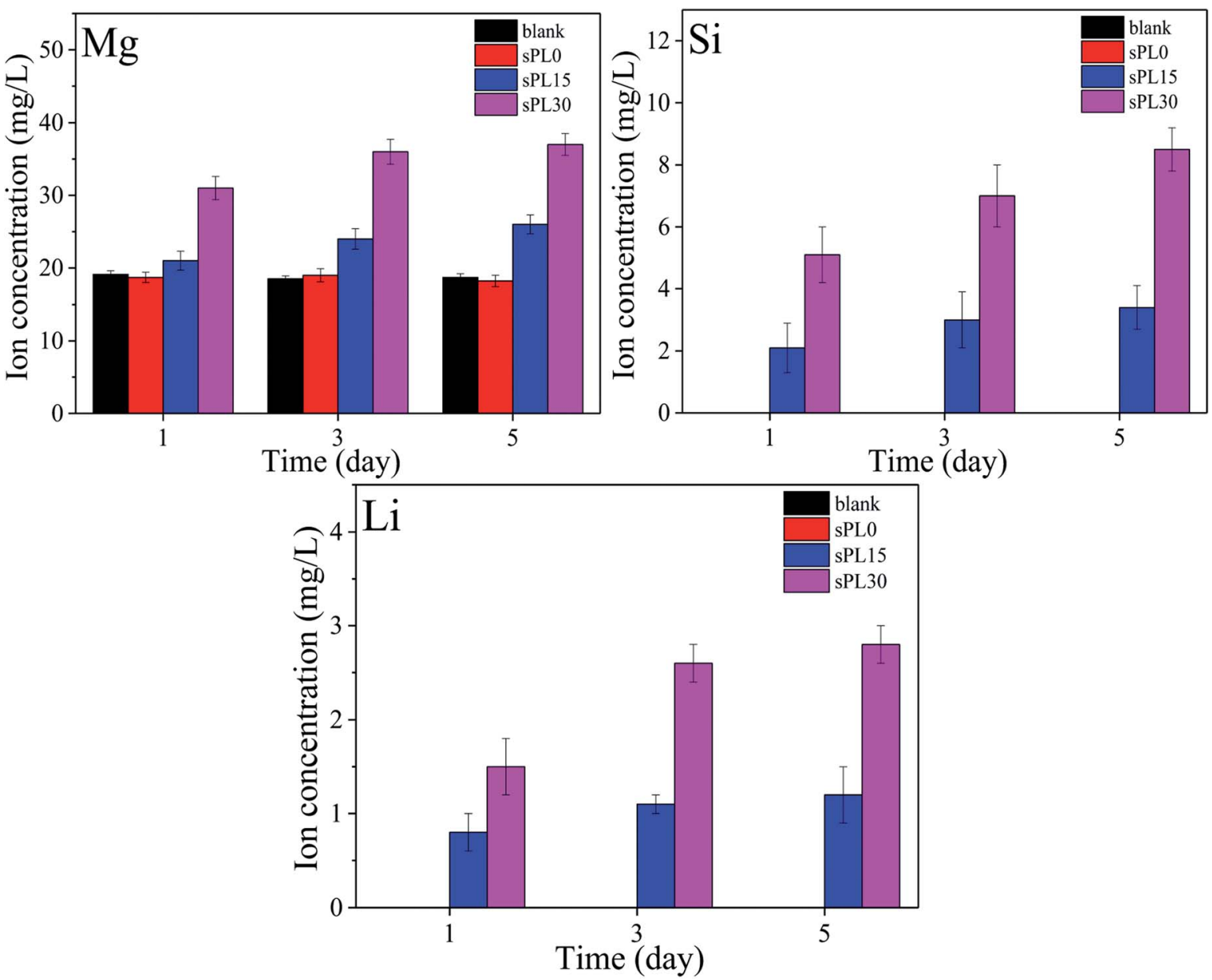

Fig. 9 Changes of concentrations of Mg, Si and Li ions in cell cultured medium with times after the MC3T3-E1 cells cultured on the samples.

is usually utilized to predict the osteogenic bioactivity of the biomaterial in vivo. ${ }^{12}$ In this study, the results showed that the mineralized apatite formed on the composites scaffolds surfaces, in which the sPL30 scaffold exhibited the best apatite mineralization ability, indicating that incorporation of LAP into PBSu significantly improved the apatite formation ability of the scaffolds, which increased with LAP content. LAP is silicate, thus the mechanism of apatite mineralization on the composites scaffolds might be similar to the apatite mineralization on silicate-based biomaterials in $\mathrm{SBF}$ (rich $\mathrm{Si}-\mathrm{OH}$ first formed on material surface in SBF, then induced $\mathrm{Ca}$ ion distribution, calcium phosphate nucleation, and ultimate apatite formation) as described in previous publications. ${ }^{5}$

Cells adhesion and spreading on the biomaterial surface are the first sequential reactions, which are crucial for subsequent cells proliferation and differentiation. ${ }^{34}$ In this study, from the SEM micrographs, the MC3T3-E1 cells adhered and spread better on SPL30 than SPL15, and SPL15 better than SPL0 scaffolds. Furthermore, from the CLSM images of the cytoskeleton of the cells on the scaffolds stained by FITC and DAPI, more cells spread and anchored better on SPL30 than SPL15, and sPL15 than SPL0 scaffolds. The results indicated that the cells adhesion and spreading on the scaffolds clearly promoted by the increase of LAP content, in which SPL30 scaffold exhibited good cytocompatibility that was favorable for cell attachment and spreading.

Generally, the OD values were used to determine the proliferation of cells on various substrates, and ideal scaffolds for bone tissue engineering should be able to stimulate cells proliferation. ${ }^{35}$ In this study, the results showed that the proliferation of the MC3T3-E1 cells on SPL30 was significantly higher than SPL15, and SPL15 was higher than SPL0 scaffolds. It can be demonstrated that the scaffolds containing LAP clearly promoted the cells proliferation, which depended on LAP content. Alkaline phosphatase (ALP) activity is an early marker of osteogensis and the increased ALP activity is often associated with the differentiation of osteoblasts..$^{35}$ In this study, the results revealed that the ALP activity of cells on SPL30 was clearly higher than SPL15, and SPL15 was higher than SPL0 scaffolds. It can be suggested that scaffolds containing LAP clearly promoted cells differentiation, which increased with LAP content.

The surface characteristics (including roughness, hydrophilicity, apatite formation and ions release, etc.) of the scaffolds play key roles in facilitating cells responses (e.g. attachment, proliferation and differentiation). ${ }^{36}$ In this study, compared 
with SPL0 scaffolds, the composites scaffolds exhibited rough and hydrophilic surfaces due to the incorporation of LAP into PBSu matrix, which increased with LAP content (sPL30 > sPL15). Therefore, the sPL30 scaffold with rough and hydrophilic surface was more favoured cells adhesion, proliferation and differentiation. The mineralized apatite on the biomaterial surface was able to adsorb serum proteins and growth factors, which then stimulated cell response (e.g. proliferation and differentiation) that was closely related to the new bone formation in vivo. ${ }^{37}$ In this study, the results demonstrated that the apatite formed on the composites scaffolds surface in SBF, which increased with LAP content (sPL30 > sPL15). Therefore, it could be suggested that the good apatite formation ability on samples surface would improve the significantly promotion of the cells responses to samples.

It has been proved that silica ( $\mathrm{Si}$ ) release from the bioactive materials (such as bioglass, bioceramics, calcium silicate, etc.) significantly stimulated the attachment, proliferation, differentiation and gene expressions of osteoblasts/BMSCs in vitro, and promoted new bone regeneration in vivo. ${ }^{38}$ Moreover, as an important element in the human body, magnesium $(\mathrm{Mg})$ is not only involved in the stabilization of all polyphosphate compounds in cells but also needed for numerous cellular functions including enzyme activity, ion channel action, metabolic pathways and signaling processes. ${ }^{39}$ Furthermore, it was reported that lithium (Li) ions exhibited stimulation effects on osteoblasts differentiation and proliferation. Moreover, lithium can up-regulate Wnt/ $\beta$-catenin signaling pathway, which plays an important role in bone tissue formation. ${ }^{40}$ LAPONITE® has been shown to degrade, resulting in the release of degradation products such as $\mathrm{Si}, \mathrm{Mg}$ and $\mathrm{Li}$ ions. In this study, the results showed that the $\mathrm{Mg}, \mathrm{Si}$ and $\mathrm{Li}$ ions could gradually release from both SPL15 and SPL30 scaffolds (dissolution of LAP) into cell culture medium with time. Therefore, compared with the SPL0 scaffold, the improvement of cells responses (e.g. proliferation and differentiation) was ascribed to the release of $\mathrm{Si}, \mathrm{Mg}$ and $\mathrm{Li}$ ions from composites scaffolds. Moreover, compared with sPL15 scaffold, the improvement of cells responses to SPL30 scaffold was ascribed to more $\mathrm{Si}, \mathrm{Mg}$ and $\mathrm{Li}$ ions released from sPL30 than sPL15 scaffold. Therefore, the promotions of cells responses (e.g. attachment, proliferation and differentiation) were ascribed to the improvements of surface characteristics of the scaffolds, including roughness, hydrophilicity, ions release and apatite formation, etc. In short, the sPL30 scaffolds clearly improved the adhesion, proliferation and differentiation of MC3T3-E1 cells, which might have a great potential for applications in bone tissue engineering.

\section{Conclusions}

In this study, novel bio-nanocomposite scaffolds were fabricated by incorporation of LAP into PBSu. The results revealed that the incorporation of LAP into PBSu was beneficial for improving the water absorption, degradability and bioactivity of the scaffolds, which were dependent on LAP content. In addition, the degradation of LAP produced the alkaline products, which neutralized acidic degradable products of $\mathrm{PBSu}$, and formed a microenvironment similar to the biological environment. Moreover, the scaffolds contained LAP clearly promoted the adhesion, proliferation and differentiation of MC3T3-E1 cells, which were dependent on LAP content. The surface characteristics (including roughness, hydrophilicity, ions release and apatite formation, etc.) of the scaffolds played key roles in facilitating cells responses (e.g. attachment, proliferation and differentiation). The sPL30 scaffolds with good cytocompatibility might have a great potential for applications in bone tissue engineering.

\section{Conflicts of interest}

There are no conflicts to declare.

\section{Acknowledgements}

The grants were from the National Natural Science Foundation of China (51502340, 81771990 and 81572194), the Major International Joint Research Project between China and Korea (81461148033), and Zhejiang Provincial Natural Science Foundation of China (LY15E020001), Medical Scientific Research Foundation of Zhejiang Province (2014PYA021).

\section{Notes and references}

1 D. M. Gibbs, C. R. Black, G. Hulsart-Billstrom, P. Shi, E. Scarpa and Oreffo, Biomaterials, 2016, 99, 16-23.

2 M. I. Carretero, Appl. Clay Sci., 2002, 21, 155-163.

3 A. K. Gaharwar, S. M. Mihaila, A. Swami, A. Patel, S. Sant, R. L. Reis, A. P. Marques, M. E. Gomes and A. Khademhosseini, Adv. Mater., 2013, 25, 3329-3336.

4 A. K. Gaharwar, P. J. Schexnailder, Q. Jin, C. J. Wu and G. Schmidt, ACS Appl. Mater. Interfaces, 2010, 2, 3119.

5 C. S. Wang, S. G. Wang, K. Li, J. P. Ju, J. P. Li, Y. X. Zhang, J. H. Li, X. Y. Liu, X. Y. Shi and Q. H. Zhao, PLoS One, 2014, 9, e99585.

6 S. G. Wang, F. Y. Zheng, Y. P. Huang, Y. T. Fang, M. W. Shen, M. F. Zhu and X. Y. Shi, ACS Appl. Mater. Interfaces, 2012, 4, 6393.

7 C. W. Chang, A. Spreeuwel, C. Zhang and S. Varghese, Soft Matter, 2010, 6, 5157-5164.

8 Y. L. Zou, Q. N. Taheri, K. Zane, M. Sadati, Q. Wei, J. Y. Liao, J. M. Fan, D. Z. Song, J. X. Liu, C. Ma, X. Y. Qu, L. Q. Chen, X. Y. Yu, Z. C. Zhang, C. Zhao, Z. Y. Zeng, R. Y. Zhang, S. J. Yan, T. T. Wu, Y. Shu, Y. S. Li, W. W. Zhang, R. R. Reid, M. J. Lee, J. M. Wolf, M. Tirrell, T. C. He, J. J. Pablo and Z. L. Deng, ACS Appl. Mater. Interfaces, 2017, 9, 15922.

9 M. Ghadiri, W. Chrzanowski, W. H. Lee and R. Rohanizadeh, RSC Adv., 2014, 4, 35332-35343.

10 C. H. Zhang, D. Cheng, T. H. Tang, X. L. Jia, Q. Cai and X. P. Yang, J. Mater. Chem. B, 2015, 3, 5300-5309.

11 W. Yu, T. W. Sun, Z. Y. Ding, C. Qi, H. K. Zhao, F. Chen, Z. M. Shi, Y. J. Zhu, D. Y. Chen and Y. H. He, J. Mater. Chem. B, 2017, 5, 1039-1052. 
12 M. Nerantzaki, M. Filippousi, G. Van Tendeloo, Z. Terzopoulou, D. Bikiaris, O. M. Goudouri, R. Detsch, A. Gruenewald and A. R. Boccaccini, eXPRESS Polym. Lett., 2015, 9, 773-789.

13 C. Gualandi, M. Soccio, M. Govoni, S. Valente, N. Lotti, A. Munari, E. Giordano, G. Pasquinelli, M. L. Focarete and J. Bioact, J. Bioact. Compat. Polym., 2012, 27, 244-264.

14 S. N. Sheikholeslami, M. Rafizadeh, F. A. Taromi and H. Shirali, Ind. Eng. Chem. Res., 2017, 56, 10343-10353.

15 M. Soccio, N. Lotti, M. Gigli, L. Finelli, M. Gazzano and A. Munari, Polym. Int., 2012, 61, 1163-1169.

16 S. Z. Fu, P. Y. Ni, B. Y. Wang, B. Y. Chu, J. R. Peng, L. Zheng, X. Zhao, F. Luo, Y. Q. Wei and Z. Y. Qian, Biomaterials, 2012, 33, 8363-8371.

17 M. Gigli, M. Fabbri, N. Lotti, R. Gamberini, B. Rimini and A. Munari, Eur. Polym. J., 2016, 75, 431-460.

18 L. Liverani, A. Piegat, A. Niemczyk, M. E. Fray and A. R. Boccaccini, Eur. Polym. J., 2016, 81, 295-306.

19 M. Soccio, N. Lotti, M. Gazzano, M. Govoni, E. Giordano and A. Munari, React. Funct. Polym., 2012, 72, 856-867.

20 M. Gigli, N. Govoni, N. Lotti, E. D. Giordano, E. D. Gazzano, M. Gazzano and A. Munari, RSC Adv., 2014, 4, 32965-32976.

21 M. Fabbri, M. Gigli, M. Costa, M. Govoni, P. Seri, N. Lotti, E. Giodrano, A. Munari, R. Gamberini, B. Rimini, G. Neretti, A. Cristofolini and C. A. Borghi, Polym. Degrad. Stab., 2015, 121, 271-279.

22 H. Chen, M. Gigli, C. Gualandi, R. Truckenmuller, N. van Blitterswijk, N. Lotti, A. Munari, M. L. Focarete and L. Moroni, Biomaterials, 2016, 76, 261-272.

23 A. A. Vassiliou, S. A. Papadimitriou, D. N. Bikiaris, G. Mattheolabakis and K. Avgoustakis, J. Controlled Release, 2010, 148, 388-395.

24 E. Liorens, H. Ibanez, L. J. Del Valle and J. Puiggali, Mater. Sci. Eng., C, 2015, 49, 472-484.

25 M. Gigli, N. Lotti, M. Gazzano, L. Finelli and A. Munari, J. Appl. Polym. Sci., 2012, 126, 686-696.
26 F. Gualandi, M. Soccio, E. Saino, M. L. Focarete, N. Lotti, A. Munari, L. Moroni and L. Visai, Soft Matter, 2012, 8, 5466-5476.

27 M. Gigli, N. Lotti, M. Gazzano, L. Finelli and A. Munari, Polym. Eng. Sci., 2013, 53, 491-501.

28 M. Fabbri, M. Gigli, R. Gamberini, N. Lotti, M. Gazzano, B. Rimini and A. Munari, Polym. Degrad. Stab., 2014, 108, 223-231.

29 V. Karageorgiou and D. Kaplan, Biomaterials, 2005, 26, 54745491.

30 D. Depan, P. K. Surya, B. Girase and R. D. Misra, Acta Biomater., 2011, 7, 2163-2175.

31 N. Su, P. L. Gao, K. Wang, J. Y. Wang, Y. Zhong and L. Ying, Biomaterials, 2017, 141, 74-85.

32 H. Yang, W. H. Dan, S. B. Xiong, Y. Kang, A. Dhinakar, J. Wu and Z. P. Gu, Acta Biomater., 2017, 47, 135-148.

33 S. L. Wu, X. M. Liu, K. W. K. Yeung, C. S. Liu and X. J. Yang, Mater. Sci. Eng., R, 2014, 80, 1-36.

34 Y. Luo, H. Shen, Y. X. Fang, Y. H. Cao, J. Huang, M. X. Zhang, J. W. Dai, X. Y. Shi and Z. J. Zhang, ACS Appl. Mater. Interfaces, 2015, 7, 6331.

35 K. L. Lin, L. G. Xia, H. Y. Li, X. Q. Jiang, H. B. Pan, Y. J. Xu, W. W. Lu, Y. F. Zhang and J. Chang, Biomaterials, 2013, 34, 10028-10042.

36 C. T. Wu and J. Chang, J. Controlled Release, 2014, 193, 282295.

37 P. S. P. Poh, D. W. Hutmacher, B. M. Holzapfel, A. K. Solanki, M. M. Stevens and M. A. Woodruff, Acta Biomater., 2016, 30, 319-333.

38 C. T. Wu, W. Fan and J. Chang, J. Mater. Chem. B, 2013, 1, 2710-2718.

39 S. Galli, Y. Naito, J. Karlsson, W. X. He, I. Miyamoto, Y. Xue, M. Andersson, K. Mustafa, A. Wemmerberg and R. Jimbo, Acta Biomater., 2014, 10, 5193-5201.

40 L. Li, X. Z. Peng, Y. B. Qin, R. C. Wang, J. L. Tang, X. Cui, T. Wang, W. L. Liu, H. B. Pan and B. Li, Sci. Rep., 2017, 7, 45204. 